\title{
ON THE $C^{1}$ NON-INTEGRABILITY OF DIFFERENTIAL SYSTEMS VIA PERIODIC ORBITS
}

\author{
JAUME LLIBRE ${ }^{1}$ AND CLÀUDIA VALLS ${ }^{2}$
}

\begin{abstract}
We go back to results of Poincaré on the multipliers of a periodic orbit for proving the $C^{1}$ non-integrability of differential systems. We apply these results to relevant systems such as the Lorenz, the Rossler and the Michelson systems, among others.
\end{abstract}

\section{Introduction And StATEMENTS of the Main RESUlts}

These last years the Ziglin's and the Morales-Ramis' theories has been used for studying the non-meromorphic integrability of a autonomous differential systems. In some sense the Ziglin's theory is a continuation of Kovalevskaya's ideas used for studying the integrability of the rigid body because it relates the non integrability of the considered system with the behavior of some of its non-equilibrium solutions as function of the complex time using the monodromy group of their variational equations. Ziglin's theory was extended to the so-called Morales-Ramis' theory which replace the study of the monodromy group of the variational equations by the study of their Galois differential group, which is easier to analyze (see [8] for more details and the references therein). But as Ziglin's theory the Morales-Ramis' theory only can study the non-existence of meromorphic first integrals.

Kovalevskaya's idea and consequently Ziglin's and Morales-Ramis' theory go back to Poincaré (see Arnold [1]), who used the multipliers of the monodromy group of the variational equations associated to periodic orbits for studying the non integrability of autonomous differential systems. The main difficulty for applying Poincaré's non integrability method to a given autonomous differential system is to find for such an equation periodic orbits having multipliers different from 1.

It seems that this result of Poincaré was forgotten in the mathematical community until that modern Russian mathematicians (specially Kozlov) have recently publish on it, see $[1,10]$.

We consider the autonomous differential system

$$
\dot{x}=f(x),
$$

where $f: U \rightarrow \mathbb{R}^{n}$ is $C^{2}, U$ is an open subset of $\mathbb{R}^{n}$ and the dot denotes the derivative with respect to the time $t$. We write its general solution as $\phi\left(t, x_{0}\right)$ with $\phi\left(0, x_{0}\right)=x_{0} \in U$ and $t$ belonging to its maximal interval of definition.

We say that the solution $\phi\left(t, x_{0}\right)$ is $T$-periodic with $T>0$ if and only if $\phi\left(T, x_{0}\right)=$ $x_{0}$ and $\phi\left(t, x_{0}\right) \neq x_{0}$ for $t \in(0, T)$. The periodic orbit associated to the periodic

1991 Mathematics Subject Classification. Primary 34C05, 34A34, 34C14.

Key words and phrases. $C^{1}$ integrability, differential systems, periodic orbits. 
solution $\phi\left(t, x_{0}\right)$ is $\gamma=\left\{\phi\left(t, x_{0}\right), t \in[0, T]\right\}$. The variational equation associated to the $T$-periodic solution $\phi\left(t, x_{0}\right)$ is

$$
\dot{M}=\left(\left.\frac{\partial f(x)}{\partial x}\right|_{x=\phi\left(t, x_{0}\right)}\right) M
$$

where $M$ is an $n \times n$ matrix. Of course $\partial f(x) / \partial x$ denotes the Jacobian matrix of $f$ with respect to $x$. The monodromy matrix associated to the $T$-periodic solution $\phi\left(t, x_{0}\right)$ is the solution $M\left(T, x_{0}\right)$ of $(2)$ satisfying that $M\left(0, x_{0}\right)$ is the identity matrix. The eigenvalues of the monodromy matrix associated to the periodic solution $\phi\left(t, x_{0}\right)$ are called the multipliers of the periodic orbit.

The following proposition and theorem go back to Poincaré (see [20]). Since we cannot find their explicit proofs in the literature, we prove them in Section 2.

Proposition 1. Let $\phi\left(t, x_{0}\right)$ be a T-periodic orbit of the $C^{2}$ differential system (1). The eigenvector tangent to the periodic orbit has associated an eigenvalue equal to 1. So the periodic orbit has at least one multiplier equal to 1.

Let $F: U \rightarrow \mathbb{R}$ be a non-constant function of class $C^{1}$ such that

$$
\nabla F(x) \cdot f(x)=0 \text {. }
$$

Then $F$ is called a first integral of $f$, because $F$ is constant on the solutions of system (1). We note that - indicates the usual inner product of $\mathbb{R}^{n}$.

Given an $n \times n$-matrix $N$ we denote its transpose by $N^{T}$. The gradient of $F$ is defined as

$$
\nabla F(x)=\left(\frac{\partial F}{\partial x_{1}}, \ldots, \frac{\partial F}{\partial x_{n}}\right)
$$

We say that two first integrals $F: U \rightarrow \mathbb{R}$ and $G: U \rightarrow \mathbb{R}$ are linearly independent if their gradients are independent in all the points of $U$ except into a set of Lebesgue measure zero.

Theorem 2. Let $f: U \rightarrow \mathbb{R}$ be the $C^{2}$ vector field associated to (1), and let $F_{k}: U \rightarrow \mathbb{R}$ a first integral for $k=1, \ldots, r$ with $r<n$. Assume that $F_{1}, \ldots, F_{r}$ are linearly independent in $U$. Let $\gamma$ be a T-periodic orbit of the vector field $f$ such that at every point $x \in \gamma$ the vectors $\nabla F_{1}(x), \ldots, \nabla F_{r}(x)$ and $f(x)$ are linearly independent. Then 1 is a multiplier of the periodic orbit $\gamma$ with multiplicity at least $r+1$.

The following two results are an immediate consequence of Theorem 2 .

Corollary 3. Consider the $C^{2}$ differential system (1). If there is a periodic orbit $\gamma$ having only $s+1$ multipliers equal to 1 , then system (1) has at most $F_{1}, \ldots, F_{s} C^{1}$ linearly independent first integrals defined in a neighborhood of $\gamma$ satisfying that the vectors $\nabla F_{1}(x), \ldots, \nabla F_{s}(x)$ and $f(x)$ are linearly independent on the points $x \in \gamma$.

Corollary 4. Under the assumptions of Corollary 3 if $s=0$, then system (1) has no $C^{1}$ first integrals $F$ defined in a neighborhood of $\gamma$ such that the vectors $\nabla F(x)$ and $f(x)$ are linearly independent on the points $x \in \gamma$.

These two corollaries give us a tool for studying the $C^{1}$ non-integrability of system (1) in a neighborhood of the periodic orbit $\gamma$. Note that Corollary 4 prevents the existence of a $C^{1}$ first integral of system (1) defined in $U$.

Using Corollary 4 we shall prove under convenient assumptions the non-existence of $C^{1}$ first integrals for systems having a zero-Hopf bifurcation, see Theorem 5 . 
Later on using Theorem 5 we shall show the non-existence of $C^{1}$ first integrals for the Lorenz system (see Theorem 6) and for the Rössler system (see Theorem 7). Finally we shall prove the non-existence of $C^{1}$ first integrals for the Michelson system (see Theorem 8), but for such a system we will not be able to apply Theorem 5.

The following four theorems are proved in section 3.

Now we present four applications of Corollary 4 showing the $C^{1}$ non-integrability of some differential systems in $\mathbb{R}^{3}$.

Theorem 5. Consider a $C^{3}$ differentiable system in $\mathbb{R}^{3}$ having the origin as a singular point with eigenvalues $\varepsilon a \pm c i$ and $\varepsilon d$. Then such a system can be written as

$$
\begin{aligned}
& \dot{x}=p(x, y, z)=\varepsilon a x-c y+\sum_{i+j+k=2} A_{i j k} x^{i} y^{j} z^{k}+O_{3}(x, y, z), \\
& \dot{y}=q(x, y, z)=c x+\varepsilon a y+\sum_{i+j+k=2} B_{i j k} x^{i} y^{j} z^{k}+O_{3}(x, y, z), \\
& \dot{z}=r(x, y, z)=\varepsilon d z+\sum_{i+j+k=2} C_{i j k} x^{i} y^{j} z^{k}+O_{3}(x, y, z),
\end{aligned}
$$

where $O_{3}(x, y, z)$ denotes the terms of order at least three in $x, y, z$. Let

$$
\begin{aligned}
& F=A_{101}+B_{011}, \\
& G=C_{020}+C_{200}, \\
& D=c\left(-4 a C_{002}+d F\right), \\
& E=D^{2}+8 a c^{2} F\left(-2 a C_{002}+d F\right) .
\end{aligned}
$$

Assume that $\left(E-D^{2}\right) /(F G)>0$ and that $(D \pm \sqrt{E}) /\left(2 c^{2} F\right) \neq 1$. Then system (3) has a limit cycle $\gamma_{\varepsilon}$ tending to the origin as $\varepsilon$ tends to zero. Moreover, there exists $\varepsilon_{0}>0$ such that for either $\varepsilon \in\left(-\varepsilon_{0}, 0\right)$ or $\varepsilon \in\left(0, \varepsilon_{0}\right)$ system $(3)$ has no $C^{1}$ first integrals $F$ defined in a neighborhood of $\gamma_{\varepsilon}$ such that the vectors $\nabla F(x, y, z)$ and $(p(x, y, z), q(x, y, z), r(x, y, z))$ are linearly independent on the points of $\gamma_{\varepsilon}$.

We should apply Theorem 5 to the Lorenz and the Rossler systems.

Theorem 6 (Lorenz system). Consider the Lorenz system

$$
\dot{x}=\sigma(y-z), \quad \dot{y}=r x-y-x z, \quad \dot{y}=-b z+x y,
$$

with $(x, y, z) \in \mathbb{R}^{3}$ and the parameters $\sigma, r, b \in \mathbb{R}$. We change the parameters $b$ and $r$ by the parameters $a$ and $c$ through

$$
\begin{aligned}
b & =-2 a \varepsilon+\frac{(c+a \varepsilon)^{2}(\sigma-1)}{c^{2}+a^{2} \varepsilon^{2}+4 a \varepsilon \sigma+2 \sigma(1+\sigma)}, \\
r & =r_{1} / r_{2}, \\
r_{1} & =\left(c^{2}+a^{2} \varepsilon^{2}\right)^{2}+\left(c^{2}(3+2 a \varepsilon)+a \varepsilon(-4+a \varepsilon(-5+2 a \varepsilon))\right) \sigma+\left(c^{2}+a \varepsilon(-4 a \varepsilon)\right) \sigma^{2}, \\
r_{2} & =c^{2}(1+2 a \varepsilon-\sigma)+a \varepsilon\left(2 a^{2} \varepsilon^{2}+4 \sigma(1+\sigma)+a(\varepsilon+7 \varepsilon \sigma)\right) .
\end{aligned}
$$

Set

$$
K=\frac{a\left(4+c^{2}+2 \sigma-2 \sigma^{2}\right)}{c^{2}+\sigma-2 \sigma^{2}+\sigma^{3}} .
$$

If $K>0$ there exists $\varepsilon_{0}>0$ such that for $\varepsilon \in\left(-\varepsilon_{0}, \varepsilon_{0}\right)$ in a neighborhood of the singular point $q=(\sqrt{b r-b}, \sqrt{b r-b}, r-1)$, the Lorenz system has no limit cycles 
if $\varepsilon<0$ and has a unique limit cycle $\gamma_{\varepsilon}$ if $\varepsilon>0$. Moreover $\gamma_{\varepsilon} \rightarrow q$ if $\varepsilon \rightarrow 0$. For $K<0$ the limit cycle $\gamma_{\varepsilon}$ exists only for $\varepsilon \in\left(-\varepsilon_{0}, 0\right)$.

If $(D \pm \sqrt{E}) /\left(2 c^{2} F\right) \neq 1$, with $D, E, F$ given in (4) with

$$
\begin{aligned}
A_{101} & =\frac{Q_{1}}{\left(2+c^{2}+2 \sigma\right) \Delta^{3 / 2} Q}+O(\varepsilon), \\
B_{011} & =\frac{Q_{2}}{\left(2+c^{2}+2 \sigma\right) \Delta^{3 / 2} Q}+O(\varepsilon), \\
C_{200} & =-\frac{Q_{3}}{\Delta^{1 / 2} Q}+O(\varepsilon), \\
C_{020} & =0, \\
C_{002} & =-\frac{Q_{4}}{\left(2+c^{2}+2 \sigma\right) \Delta^{3 / 2} Q}+O(\varepsilon),
\end{aligned}
$$

where

$$
\begin{aligned}
& \Delta=\frac{c^{2}\left(c^{2}+(1+\sigma)^{2}\right)}{c^{2}+2 \sigma(1+\sigma)}, \\
& Q=c^{6}+12 c^{2} \sigma^{2}(1+\sigma)^{2}+4 \sigma^{2}(1+\sigma)^{4}+4 c^{4} \sigma(1+2 \sigma), \\
& Q_{1}=c^{4}(1+\sigma)\left(c^{2}+(1+\sigma)^{2}\right)\left(c^{4}+8 c^{2} \sigma+4 \sigma(1+\sigma)^{2}\right), \\
& Q_{2}=c^{4}(-1+\sigma)\left(c^{2}+(1+\sigma)^{2}\right)\left(c^{4}-2 c^{2} \sigma(1+\sigma)-4 \sigma(1+\sigma)^{3}\right), \\
& Q_{3}=\sigma^{2}\left(2+c^{2}+2 \sigma\right)\left(c^{2}+\sigma(1+\sigma)^{2}\right), \\
& Q_{4}=c^{4} \sigma\left(c^{2}-2(\sigma-2)(1+\sigma)\right)\left(c^{2}+(1+\sigma)^{2}\right)^{2},
\end{aligned}
$$

then the Lorenz system for $\varepsilon \in\left(0, \varepsilon_{0}\right)$ when $K>0$, and for $\varepsilon \in\left(-\varepsilon_{0}, 0\right)$ when $K<0$ has no $C^{1}$ first integrals $F(x, y, z)$ defined in a neighborhood of the zeroHopf periodic orbit $\gamma_{\varepsilon}$ satisfying that $\nabla F(x, y, z)$ and $(\sigma(y-z), r x-y-x z,-b z+x y)$ are linearly independent on the points of $\gamma_{\varepsilon}$.

The Lorenz system (5) was defined in [12]. This system has been intensively studied from the point of view of integrability using different integrability theories, and in particular it has been studied its Darboux integrability and its analytic integrability (for example, see $[3,5,6,7,9,16,22,23,24,25,26,27]$ ), but never from the view point of the $C^{1}$ integrability.

The Rossler system (see (6) below) was obtained in [21]. It is a well-known dynamical model which has been intensively investigated mainly with respect the notion of dynamical chaos.

Theorem 7 (Rossler system). Consider the Rossler system

$$
\dot{x}=-(y+z), \quad \dot{y}=x+a y, \quad \dot{y}=b-c z+x z,
$$

with $(x, y, z) \in \mathbb{R}^{3}$ and the parameters $a, b, c \in \mathbb{R}$. We change the parameters $a, b$ and $c$ by the parameters $a, u$ and $v$ through

$$
\begin{aligned}
& b=-\frac{b_{1} b_{2}}{\left(-1+(a-\varepsilon u)^{2}+v^{2}\right)^{2}}, \\
& c=-a-2 \varepsilon u+a \varepsilon^{2} u^{2}+a v^{2}+\frac{(a-2 \varepsilon u)\left(1+a^{2}-2 a \varepsilon u\right)}{-1+(a-\varepsilon u)^{2}+v^{2}}, \\
& b_{1}=(-1+\varepsilon u(a-\varepsilon u))^{2}+\left(-2+a^{2}-2 a \varepsilon u+2 \varepsilon^{2} u^{2}\right) v^{2}+v^{4}, \\
& b_{2}=2 a^{2} \varepsilon u-a\left(1+4 \varepsilon^{2} u^{2}\right)+2 \varepsilon u\left(\varepsilon^{2} u^{2}+v^{2}\right) .
\end{aligned}
$$


Set

$$
L=a u\left(-2+a^{2}+v^{2}\right) \Lambda_{2}
$$

with

$$
\Lambda_{2}=-2+4 a^{2}-4 a^{4}+a^{6}+5 v^{2}-8 a^{2} v^{2}+3 a^{4} v^{2}-4 v^{4}+3 a^{2} v^{4}+v^{6} .
$$

If $L>0$ there exists $\varepsilon_{0}>0$ sufficiently small such that for $\varepsilon \in\left(-\varepsilon_{0}, \varepsilon_{0}\right)$ in a neighborhood of the singular point

$$
s=\left(\frac{c+\sqrt{c^{2}-4 a b}}{2},-\frac{c+\sqrt{c^{2}-4 a b}}{2 a}, \frac{c+\sqrt{c^{2}-4 a b}}{2 a}\right),
$$

the Rossler system (6) has no limit cycles if $\epsilon<0$ and has a unique limit cycle $\gamma_{\varepsilon}$ if $\epsilon>0$ that tends to $q$ when $\epsilon \rightarrow 0$. For $L<0$ the limit cycle only exists for $\varepsilon \in\left(-\varepsilon_{0}, 0\right)$.

If $(D \pm \sqrt{E}) /\left(2 c^{2} F\right) \neq 1$, with $D, E, F$ given in (4) with

$$
\begin{aligned}
& A_{101}=-\frac{a\left(\Delta_{1}-1\right)\left(v^{2}+\Delta_{1}\left(a^{2}+\left(-2+a^{2}\right) v^{2}+v^{4}\right)\right)}{\Delta_{1}\left(1+\left(-2+a^{2}\right) v^{2}+v^{4}\right) \Delta_{2}}+O(\varepsilon), \\
& B_{011}=\frac{a\left(\Delta_{1}-1\right)^{3}\left(a^{4}-v^{2}+v^{4}+2 a^{2}\left(-1+v^{2}\right)\right)}{\Delta_{1}\left(1+\left(-2+a^{2}\right) v^{2}+v^{4}\right) \Delta_{2}}+O(\varepsilon), \\
& C_{200}=\frac{a \Delta_{1}\left(1+\left(-2+a^{2}\right) v^{2}+v^{4}\right)}{v^{2}\left(1+\left(-2+a^{2}\right) v^{2}+v^{4}\right) \Delta_{2}}+O(\varepsilon), \\
& C_{020}=0, \\
& C_{002}=\frac{a\left(\Delta_{1}-1\right)}{\Delta_{2}}+O(\varepsilon),
\end{aligned}
$$

where

$$
\begin{aligned}
& \Delta_{1}=a^{2}+v^{2} \\
& \Delta_{2}=v^{2}+\left(-2+a^{2}+v^{2}\right)\left(a^{4}+v^{4}+2 a^{2}\left(-1+v^{2}\right)\right), \\
& \Lambda_{1}=2 u-4 u v^{2}+2 a^{2} u v^{2}+2 u v^{4}+2 w-4 a^{2} w+4 a^{4} w-a^{6} w- \\
& 5 v^{2} w+8 a^{2} v^{2} w-3 a^{4} v^{2} w+4 v^{4} w-3 a^{2} v^{4} w-v^{6} w \\
& \Lambda_{3}=1-2 v^{2}+a^{2} v^{2}+v^{4} \\
& \Lambda_{4}=4 a^{2}-4 a^{4}+a^{6}+v^{2}-6 a^{2} v^{2}+3 a^{4} v^{2}-2 v^{4}+3 a^{2} v^{4}+v^{6},
\end{aligned}
$$

then the Rossler system for $\varepsilon \in\left(0, \varepsilon_{0}\right)$ when $L>0$, and for $\varepsilon \in\left(-\varepsilon_{0}, 0\right)$ when $L<0$ has no $C^{1}$ first integrals $F(x, y, z)$ defined in a neighborhood of the zero-Hopf periodic orbit $\gamma_{\varepsilon}$ satisfying that $\nabla F(x, y, z)$ and $(-(y+z), x+a y, b-c z+x z)$ are linearly independent on the points of $\gamma_{\varepsilon}$.

The Rossler system has been studied in [17] from the viewpoint of the Darbouxian integrability and in [14] from the viewpoint of the analytic integrability but never from the view point of the $C^{1}$ integrability.

Note that Theorem 5 cannot be applied to the Michelson system defined in the next theorem. 
Theorem 8 (Michelson system). Consider the Michelson system

$$
\dot{x}=y, \quad \dot{y}=z, \quad \dot{z}=c^{2}-y-\frac{x^{2}}{2},
$$

with $(x, y, z) \in \mathbb{R}^{3}$ and the parameter $c \in \mathbb{R}$. The Michelson system for $c>0$ sufficiently small has no $C^{1}$ first integrals $F(x, y, z)$ defined in a neighborhood of the zero-Hopf periodic orbit $\gamma$ satisfying that $\nabla F(x, y, z)$ and $\left(y, z, c^{2}-y-\frac{x^{2}}{2}\right)$ are linearly independent on the points of $\gamma$.

The Michelson system has been studied in [15] from the viewpoint of the analytic and Darboux integrability but never from the view point of the $C^{1}$ integrability.

\section{Proof of Proposition 1 And Theorem 2}

Proof of Proposition 1. Let $\phi\left(t, x_{0}\right)$ with $t \in \mathbb{R}$ and $x_{0} \in U$ be a $T$-periodic solution of the autonomous differential system (1). Clearly we have that

$$
\phi\left(\tau, \phi\left(t, x_{0}\right)\right)=\phi\left(t+\tau, x_{0}\right) .
$$

Differentiating (8) with respect to $t$ and setting $t=0$ and $\tau=T$ we get

$$
\frac{\partial \phi}{\partial x}\left(T, x_{0}\right) \dot{\phi}\left(0, x_{0}\right)=\dot{\phi}\left(T, x_{0}\right) .
$$

Since $\phi\left(T, x_{0}\right)=x_{0}$ we get that $\dot{\phi}\left(T, x_{0}\right)=f\left(\phi\left(T, x_{0}\right)\right)=f\left(x_{0}\right)$. In a similar way we have that $\dot{\phi}\left(0, x_{0}\right)=f\left(x_{0}\right)$, and thus we can rewrite (9) into the form

$$
\frac{\partial \phi}{\partial x}\left(T, x_{0}\right) f\left(x_{0}\right)=f\left(x_{0}\right) .
$$

Note that differentiating with respect to $x$ equation (1) we have that

$$
\frac{\partial}{\partial x} \frac{d \phi(t, x)}{d t}=\frac{\partial f(\phi(t, x))}{\partial x} \frac{\partial \phi(t, x)}{\partial x} .
$$

By the Schwartz's lemma we can rewrite the above equation as

$$
\frac{d}{d t} \frac{\partial \phi(t, x)}{\partial x}=\frac{\partial f(\phi(t, x))}{\partial x} \frac{\partial \phi(t, x)}{\partial x}
$$

which implies that $\frac{\partial \phi}{\partial x}(t, x)$ is a solution of $(2)$. Note that since $\phi(0, x)=x$, taking derivative with respect to $x$ we obtain that $\frac{\partial \phi(t, x)}{\partial x}=\mathrm{Id}$. Thus, $\frac{\partial \phi}{\partial x}\left(T, x_{0}\right)$ is the monodromy matrix associated to $\phi\left(t, x_{0}\right)$. It follows from $(10)$ that $\frac{\partial \phi}{\partial x}\left(T, x_{0}\right)$ has 1 as an eigenvalue with eigenvector $f\left(x_{0}\right) \neq 0$ because $x_{0}$ is not an equilibrium point. Furthermore, since $f\left(x_{0}\right)=\dot{\phi}\left(0, x_{0}\right)$ we get that the eigenvector $f\left(x_{0}\right)$ is tangent to the periodic orbit at the point $x_{0}$. This completes the proof of the proposition.

Proof of Theorem 2. Since for each $k=1, \ldots, r, F_{k}$ is a first integral of system (1), we have that $F_{k}(\phi(t, x))=F_{k}(x)$. Differentiating this relation with respect to $x$ we get

$$
\nabla F_{k}(\phi(t, x)) \frac{\partial \phi(t, x)}{\partial x}=\nabla F_{k}(x)
$$


Then taking $t=T$ and $x=x_{0}$ in the previous equality, and since $\phi\left(T, x_{0}\right)=x_{0}$, we obtain

or equivalently,

$$
\nabla F_{k}\left(x_{0}\right) \frac{\partial \phi\left(T, x_{0}\right)}{\partial x}=\nabla F_{k}\left(x_{0}\right)
$$

$$
\left(\frac{\partial \phi\left(T, x_{0}\right)}{\partial x}\right)^{T} \nabla F_{k}\left(x_{0}\right)=\nabla F_{k}\left(x_{0}\right)
$$

Therefore, $\nabla F_{k}\left(x_{0}\right)$ is an eigenvector of $\left(\frac{\partial \phi\left(T, x_{0}\right)}{\partial x}\right)^{T}$ with eigenvalue 1 . On the other hand from the proof of Proposition 1 we know that

$$
\frac{\partial \phi\left(T, x_{0}\right)}{\partial x} f\left(x_{0}\right)=f\left(x_{0}\right)
$$

For ending the proof since by assumption the vectors $\nabla F_{1}\left(x_{0}\right), \ldots, \nabla F_{r}(x)$ and $f\left(x_{0}\right)$ are linearly independent on the points of the periodic orbit $\gamma$, there at at least $r+1$ multipliers for the monodromy matrix equal to 1 .

\section{Proof of Theorems 5, 6, 7 And 8}

The proof of Theorem 5 is an immediate consequence of Corollary 4 and the following theorem (together with its proof which is given in [11]).

Theorem 9. The following statements hold for system (3).

(a) If $\left(E-D^{2}\right) /(F G)>0$ then the differential system (3) has a limit cycle $\gamma_{\varepsilon}$ tending to the origin as $\varepsilon \rightarrow 0$.

(b) The multipliers of the limit cycle $\gamma_{\varepsilon}$ are 1 and $(D \pm \sqrt{E}) /\left(2 c^{2} F\right)$.

Proof of Theorem 6 . The part of the proof of Theorem 6 concerning the existence of $\gamma_{\epsilon}$ follows from Theorem 2 in [11]. Computing the eigenvalues at the singular point $q$ and using Theorem 5 together with Corollary 4 the proof follows easily.

Proof of Theorem 7. The part of the proof of Theorem 7 concerning the existence of $\gamma_{\epsilon}$ follows from Theorem 3 in [11]. Computing the eigenvalues at the singular point $s$ and using Theorem 5 together with Corollary 4 the proof follows easily.

The Michelson system (7) was obtained by Michelson [19] in the study of the travelling wave solutions of the Kuramoto-Sivashinsky equation. It is well-known that system (7) is reversible with respect to the involution $R(x, y, z)=(-x, y,-z)$ and is volume-preserving under the flow of the system. It is easy to check that system (7) has two finite singularities

$$
p_{1}=(-\sqrt{2} c, 0,0) \text { and } p_{2}=(\sqrt{2} c, 0,0)
$$

for $c \neq 0$ which are both saddle-foci. The singular point $p_{1}$ has a 2-dimensional stable manifold and $p_{2}$ has a 2-dimensional unstable manifold. Note that when $c=0$ the Michelson system has a unique singular point at the origin with eigenvalues 0 , $\pm i$. In [18] it is proved that for $c>0$ sufficiently small the Michelson system (7) has a Hopf-zero bifurcation at the origin for $c=0$. Here we shall reproduce the short proof of [18] because it is necessary for proving our result:

To prove Theorem 8 we shall need the following result essentially due to Malkin (1956) and to Roseau (1966) (see [4]). In [2] it is given a new and shorter proof. 
Theorem 10 (Perturbations of an isochronous open set). Consider a differential system

$$
\dot{x}=F_{0}(t, x)+\varepsilon F_{1}(t, x)+\varepsilon^{2} F_{2}(t, x, \varepsilon), \quad(t, x, \varepsilon) \in \mathbb{R} \times \Omega \times\left(-\varepsilon_{0}, \varepsilon_{0}\right),
$$

where $\Omega$ is an open subset of $\mathbb{R}^{n}$, and $F_{0}, F_{1}$ and $F_{2}$ are $C^{2}$ smooth and $T$-periodic in the time $t$. Let $x(t, z)$ be a solution of (11) when $\varepsilon=0$ such that $x(0, z)=z$. Denote by $M_{z}(t)$ the fundamental solution matrix of the variational equation

$$
\dot{y}=D_{x} F_{0}(t, x(t, z)) y,
$$

such that $M_{z}(0)=I d$. Assume that there exists an open and bounded subset $V$ with its closure $\operatorname{cl}(V) \subset \Omega$ such that for each $z \in \operatorname{cl}(V)$, the solution $x(t, z)$ is $T$-periodic. If $a \in V$ is a zero of the map $F: \operatorname{cl}(V) \rightarrow \mathbb{R}^{n}$ defined by

$$
F(z)=\int_{0}^{T} M_{z}^{-1}(t) F_{1}(t, x(t, z)) d t
$$

and $\operatorname{det}\left(D_{z} F(a)\right) \neq 0$, then for $|\varepsilon|>0$ sufficiently small system (11) has a $T$ periodic solution $\phi(t, \varepsilon)$ such that $\phi(0, \varepsilon) \rightarrow a$ as $\varepsilon \rightarrow 0$. Moreover the periodic solution $\phi(t, \varepsilon)$ has the same stability type than the singular point at the origin of the linear differential system $\dot{y}=\left(D_{z} F(a)\right) y$ if this singular point is hyperbolic.

Proof of Theorem 8 . For any $\varepsilon \neq 0$ we take the change of variables

$$
x=\varepsilon \bar{x}, \quad y=\varepsilon \bar{y}, \quad z=\varepsilon \bar{z} \quad \text { and } \quad c=\varepsilon d .
$$

Then the Michelson system (7) becomes

$$
\dot{x}=y, \quad \dot{y}=z, \quad \dot{z}=-y+\varepsilon d^{2}-\varepsilon \frac{x^{2}}{2},
$$

where we still use $x, y, z$ instead of $\bar{x}, \bar{y}, \bar{z}$. Now doing the change of variables

$$
x=x, \quad y=r \sin \theta \text { and } z=r \cos \theta,
$$

system (13) goes over to

$$
\dot{x}=r \sin \theta, \quad \dot{r}=\frac{\varepsilon}{2}\left(2 d^{2}-x^{2}\right) \cos \theta, \quad \dot{\theta}=1-\frac{\varepsilon}{2 r}\left(2 d^{2}-x^{2}\right) \sin \theta .
$$

This system can be written as

$$
\begin{aligned}
& \frac{d x}{d \theta}=r \sin \theta+\frac{\varepsilon}{2}\left(2 d^{2}-x^{2}\right) \sin ^{2} \theta+\varepsilon^{2} f_{1}(\theta, r, \varepsilon), \\
& \frac{d r}{d \theta}=\frac{\varepsilon}{2}\left(2 d^{2}-x^{2}\right) \cos \theta+\varepsilon^{2} f_{2}(\theta, r, \varepsilon),
\end{aligned}
$$

where $f_{1}$ and $f_{2}$ are analytic functions in their variables.

For any given $x_{0}$ and $r_{0}$, system (15) in $\varepsilon=0$ has the $2 \pi$-periodic solution

$$
x(\theta)=r_{0}+x_{0}-r_{0} \cos \theta, \quad r(\theta)=r_{0},
$$

check such that $x(0)=x_{0}$ and $r(0)=r_{0}$. It is easy to see that the variational equation of (15) on $\varepsilon=0$ along the solution (16) is

$$
\left(\begin{array}{l}
\frac{d y_{1}}{d \theta} \\
\frac{d y_{2}}{d \theta}
\end{array}\right)=\left(\begin{array}{cc}
0 & \sin \theta \\
0 & 0
\end{array}\right)\left(\begin{array}{l}
y_{1} \\
y_{2}
\end{array}\right) .
$$


It has the fundamental matrix solution

$$
M=\left(\begin{array}{cc}
1 & 1-\cos \theta \\
0 & 1
\end{array}\right)
$$

which is independent of the initial condition $\left(x_{0}, r_{0}\right)$. Using Theorem 10 we have

$$
F\left(x_{0}, r_{0}\right)=\left.\frac{1}{2} \int_{0}^{2 \pi} M^{-1}\left(\begin{array}{c}
\left(2 d^{2}-x^{2}\right) \sin ^{2} \theta \\
\left(2 d^{2}-x^{2}\right) \cos \theta
\end{array}\right)\right|_{(16)} d \theta .
$$

Then $F\left(x_{0}, r_{0}\right)=\left(g_{1}\left(x_{0}, r_{0}\right), g_{2}\left(x_{0}, r_{0}\right)\right)$ with

$$
g_{1}\left(x_{0}, r_{0}\right)=\frac{1}{4}\left(4 d^{2}-5 r_{0}^{2}-6 r_{0} x_{0}-2 x_{0}^{2}\right), \quad g_{2}\left(x_{0}, r_{0}\right)=\frac{1}{2} r_{0}\left(x_{0}+r_{0}\right) .
$$

Solving $F\left(x_{0}, r_{0}\right)=0$ we get that it has a unique non-trivial solution $x_{0}=-2 d$ and $r_{0}=2 d$. The eigenvalues of the Jacobian matrix on this solution are

$$
\lambda_{1}=-\frac{d}{2}(1+\sqrt{5}) \neq 1 \quad \text { and } \quad \lambda_{2}=-\frac{d}{2}(1-\sqrt{5}) \neq 1,
$$

taking $d \neq 2 /(1 \pm \sqrt{5})$. Therefore, by Theorem 10, the multipliers of this zero-Hopf periodic orbit $\gamma$ are

$$
1, \quad-\frac{d}{2}(1+\sqrt{5}) \neq 1 \quad \text { and } \quad-\frac{d}{2}(1+\sqrt{5}) \neq 1
$$

if $d \neq 2 /(1 \pm \sqrt{5})$. Consequently, by Corollary 4 system $(7)$ has no $C^{1}$ first integrals $F$ defined in a neighborhood of $\gamma$ satisfying that

$$
\nabla F(x, y, z) \text { and }\left(y, z, c^{2}-y-\frac{x^{2}}{2}\right)
$$

are linearly independent on the points of $\gamma$.

\section{ACKNOWLEDGEMENTS}

The first author has been supported by the grants MCYT/FEDER numbers MTM2008-03437 and CIRIT 2009SGR-410. The second author has been partially supported by FCT through CAMGDS, Lisbon.

\section{REFERENCES}

1. V.I. Arnold, Forgotten and neglected theories of Poincaré, Russian Math. Surveys 61 No. 1 (2006), 1-18.

2. A. Buica, J.P. Francoise And J. Llibre, Periodic solutions of nonlinear periodic differential systems with a small parameter, Commun. Pure Appl. Anal. 6 (2007), 103-111.

3. L. Cairó And D. HuA, Comments on: "integrals of motion for the Lorenz system", J. Math. Phys. 34 (1993), 4370-4371.

4. J.P. Francoise, Oscillations em biologie: Analyse qualitative et modèles, Collection: Mathématiques et Applications 46, Springer Verlag 2005.

5. H.J. Giacomini, C.E. Repetto And O.P. Zandron, Integrals of motion for threedimensional non-Hamiltonian dynamical systems, J. Phys. A: Math. Gen. 24 (1991), 45674574.

6. A. GoRIELY, Integrability, partial integrability, and nonintegrability for systems of ordinary differential equations, J. Math. Phys. 37 (1996), 1871-1893.

7. N. Gupta, Integrals of motion for the Lorenz system, J. Math. Phys. 34 (1993), 801-804.

8. J.J. Morales-Ruiz, Differential Galois Theory and non-integrability of Hamiltonian systems, Progress in Math. Vol. 178, Birkhauser, Verlag, Basel, 1999.

9. M. Kús, Integrals of motion for the Lorenz system, J. Phys. A: Math. Gen. 16 (1983), 689-691.

10. V.V. KOzLOV, Integrability and non-integrability in Hamiltonian mechanics, Russian Math. Surveys 38 No. 1 (1983), 1-76. 
11. J. Llibre, C.A. Buzzi And P.R. Da Silva, 3-dimensional hopf bifurcation via averaging theorem, Discrete Contin. Dyn. Syst. 17 (2007), 529-540.

12. E.N. Lorenz, Deterministic nonperiodic flow, J. Atmos. Sci. 20 (1963), 130-141.

13. J. Llibre AND C. VAlls, Formal and analytic integrability of the Lorenz system, J. Phys. A 38 (2005), 2681-2686.

14. J. Llibre And C. Valls, Formal and analytic integrability of the Rossler system, Internat. J. Bifur. Chaos Appl. Sci. Engrg. 17 (2007), 3289-3293.

15. J. Llibre AND C. VALLS, The Michelson system is neither global analytic, nor Darboux integrable, preprint.

16. J. Llibre And X. Zhang, Invariant algebraic surfaces of the Lorenz systems, J. Math. Phys. 43 (2002), 7613-7635.

17. J. Llibre AND X. Zhang, Darboux integrability for the Rossler system, Internat. J. Bifur. Chaos Appl. Sci. Engrg. 12 (2002), 421-428.

18. J. Llibre And X. Zhang, On the Hopf-zero bifurcation of the Michelson system, preprint (2009).

19. D. Michelson, Steady solutions for the Kuramoto-Sivashinsky equation, Physica D 19 (1986), 89-111. Birkhauser, Verlag, Basel, 1999.

20. H. Poincaré, Sur l'intégration des équations différentielles du premier ordre et du premier degré $I$ and II, Rendiconti del circolo matematico di Palermo 5 (1891), 161-191; 11 (1897), 193-239.

21. E. O. Rossler, An equation for continuous chaos, Phys. Lett. A 57 (1976), 397-398.

22. F. SCHWARZ, An algorithm for determining polynomial first integrals of autonomous systems of ordinary differential equations, J. Symbol. Comput. 1 (1985), 229-233.

23. H. Segur, Soliton and the inverse scattering transform, Topics in Ocean Physics, edited by A. R. Osborne and P. Malanotte Rizzoli, North-Holland, Amsterdam, 1982, pp 235-277.

24. W.H. SteEs, Continuous symmetries of the Lorenz model and the Rikitake two-disc dynamo system, J. Phys. A: Math. Gen. 15 (1982), 389-390.

25. J.M. Strelcyn and S. Wojciechowski, A method of finding integrals for three-dimensional systems, Phys. Lett. A 133 (1988), 207-212.

26. P. Swinnerton-Dyer, The invariant algebraic surfaces of the Lorenz system, Math. Proc. Cambridge Philos. Soc. 132 (2002), 385-393.

27. X. Zhang, Exponential factors and Darbouxian first integrals of the Lorenz system, J. Math. Phys. 43 (2002), 4987-5001.

1 Departament de Matemàtiques, Universitat Autònoma de Barcelona, 08193 Bellaterra, Barcelona, Catalonia, Spain

E-mail address: jllibre@mat.uab.cat

2 Departamento de Matemática, Instituto Superior Técnico, Av. Rovisco Pais 1049001, Lisboa, Portugal

E-mail address: cvalls@math.ist.utl.pt 